\title{
La aplicación de los códigos Data Matrix (DM) en el registro y la catalogación arqueológica
}

\author{
Xavier Roda Gilabert, ${ }^{1}$ Sofia C. Samper Carro, ${ }^{2}$ Rafael Mora Torcal, ${ }^{1}$ \\ Paloma González Marcén, ${ }^{1}$ Jorge Martínez Moreno ${ }^{1}$
}

Recibido: 18-10-2012

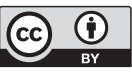

Aceptado: 15-12-2012

\section{Resumen}

El registro e inventariado de los materiales arqueológicos es una parte esencial de la investigación arqueológica. Durante el trabajo de laboratorio, cada ítem es identificado individualmente. Esta tarea se realiza generalmente a mano, lo que facilita la pérdida de información debido a errores humanos. De este modo, números erróneos, ortografía ilegible o la desaparición del identificador de los artefactos son comunes. Ante la ausencia de un método fiable y duradero de marcado de los objetos, una parte importante del trabajo en los museos consiste en el re-inventariado de las colecciones. En este trabajo se presenta la utilización de los códigos Data Matrix (DM) para el catalogado del material arqueológico. Su uso en los yacimientos de Roca dels Bous y la Cova Gran de Santa Linya (Lleida, Espańa) evidencia que la aplicación de esta técnica facilita el almacenaje y preservación de las colecciones arqueológicas.

Palabras clave: códigos Datamatrix (DM); catalogado de artefactos; gestión de colecciones; contenidos multimedia.

Resum. L'aplicació dels codis Datamatrix (DM) en el registre i catalogació arqueològica

El registre i inventari del material arqueològic es una part essencial de la recerca arqueològica. Al llarg del treball de laboratori cada objecte és identificat individualment. Aquesta tasca es realitza generalment a mà, fet que facilita la pèrdua d'informació a causa d'errors humans. D'aquesta manera números erronis, ortografia illegible o la desaparició de l'identificador són molt comuns. Davant l'absència d'un mètode fiable de marcatge, una part important del treball als museus consisteix en el reinventariat de les col-leccions. En aquest treball es presenta la utilització dels codis Datamatrix (DM) en el catalogat del material arqueològic. El seu ús als jaciments de Roca dels Bous i la Cova Gran de Santa Linya (Lleida, Espanya) evidencia que l'aplicació d'aquesta nova tècnica facilita l'emmagatzematge i la preservació de col-leccions arqueològiques.

Paraules clau: codis Datamatrix (DM); catalogat d'artefactes; gestió de col-leccions; continguts multimèdia.

1. Centre d'Estudis del Patrimoni Arqueològic de la Prehistòria (CEPAP-UAB). Universitat Autònoma de Barcelona. Facultat de Lletres. 08193 Bellaterra (Cerdanyola del Vallès).

javier.roda@uab.cat; rafael.mora@uab.cat; paloma.gonzalez@uab.cat; jorge.martinez@uab.cat

2. College of Asia \& the Pacific. H.C. Coombs Building. Department of Archaeology \& Natural History. The Australian National University. 0200 ACT.

sofia.samper@anu.edu.au 
Abstract. DataMatrix codes' (DM) application on the archaeological record

The recording and inventory of archaeological material is a key point in archaeology. During laboratory duties, each item is individually identified and labelled. This task is usually by hand, which facilitates the information loss due to human error. Thus wrong numbers, misspelling or id loss are common. The lack of a reliable method of marking leads to allocate most of museum tasks to the relabelling of collections. In this paper we present the application of DataMatrix (DM) codes for archaeological labelling. The application of DM in Roca dels Bous and Cova Gran Santa Linya (Lleida Spain) rockshelters has shown that this technique facilitates the storage and preservation of the archaeological collections.

Keywords: Datamatrix codes (DM); artefact labelling; collections management; multimedia contents.

Roda Gilabert, Xavier; Samper Carro, Sofia C.; Mora Torcal, Rafael; González MarCÉn, Paloma; Martínez Moreno, J. «La aplicación de los códigos Data Matrix (DM) en el registro y la catalogación arqueológica». Treballs d'Arqueologia, 2014, núm. 20, p. 95-107.

DOI: $10.5565 / \mathrm{rev} / \mathrm{tda} .49$

\section{Introducción}

El registro del material arqueológico es una parte esencial del trabajo en la excavación arqueológica (Renfrew \& Banh, 1991; Laplace 1971). El inventariado individualizado de los objetos es una práctica habitual en las excavaciones prehistóricas, y cada vez más común en yacimientos históricos. Gracias a esta identificación singularizada, un artefacto se puede poner en relación con el resto de objetos, con la unidad arqueológica y con el yacimiento arqueológico al que pertenecen. La identificación singularizada permite contextualizar los objetos, facilitando la documentación de patrones espaciales y la identificación de los procesos de formación del yacimiento.

El marcado de los objetos arqueológicos es una tarea que tradicionalmente se ha realizado a mano, implicando una gran inversión de recursos - tiempo y trabajo- $y$, frecuentemente, dando lugar a errores humanos. En los últimos años se ha ensayado la aplicación de sistemas de identificación digitales en el trabajo de campo. Entre ellos podemos citar el uso códigos de barras en yacimientos como Roc de Marsal (Dibble et al., 2007) o Atapuerca (Canals y Guerra Rodríguez, 2011). Alternativamente, en este artículo presentamos una implementación tecnológica que consiste en la aplicación de los códigos Data Matrix (DM) para el catalogado del material arqueológico. Esta sistemática se ejemplifica a partir de su uso en los yacimientos de Roca dels Bous y la Cova Gran de Santa Linya (Lleida, España), en los cuales esta metodología ha sido empleada a lo largo de los últimos cinco años (Martínez-Moreno et al., 2011). Esta aplicación supone una importante mejora en la gestión y catalogación del registro arqueológico, que beneficia al tratamiento de los artefactos durante las rutinas del trabajo de campo y laboratorio. 
Xavier Roda Gilabert, Sofia C. Samper Carro, Rafael Mora Torcal, Paloma González Marcén, Jorge Martínez Moreno
La aplicación de los códigos Data Matrix (DM) en el registro y la catalogación arqueológica

\section{Los códigos Data Matrix (DM)}

Los códigos Data Matrix (DM) fueron desarrollados en los ańos ochenta por RVSI Acuity Cimatrix Inc. Corporation (Nashua, US) y en la actualidad son de dominio público y están protegidos por el estándar ISO//EC16022 que garantiza su acceso libre y gratuito. Dentro de esta categoría se han desarrollado diferentes tipos de códigos, como QR-codes, Aztec codes, Semadode, idcDMatrix. (http:// en.wikipedia.org/wiki/Data_Matrix). Los DM pueden contener gran cantidad de información y su uso se ha extendido a las zonas industrializadas aplicándose a la logística, el servicio postal, la industria médica o el control de calidad de pro- ductos industriales. A esto podemos ańadir su extensión al ámbito del marketing a través del uso de dispositivos móviles tales como teléfonos o tabletas.

Estos códigos tienen una estructura bidimensional que consiste en una cuadrícula de celdas que almacenan datos alfanuméricos. Habitualmente son impresos en formato cuadrado donde cada cuadro representa un bit. Este hecho los diferencia de los códigos de barras donde la información es encriptada entre el ratio de las barras y la distancia de separación de las mismas. La cantidad de información que pueden contener depende del tamańo de los mismos (tabla 1).

Tabla 1. Tamaños de los códigos DM y capacidad numérica o alfanumérica máxima que pueden contener

\begin{tabular}{ccc}
\hline & Datos & \\
\hline Tamańo & Numéricos & Alfanuméricos \\
$3 \times 3 \mathrm{~mm}$ & 10 & 6 \\
$3,5 \times 3,5 \mathrm{~mm}$ & 16 & 10 \\
$5 \times 5 \mathrm{~mm}$ & 34 & 24 \\
\hline
\end{tabular}

Es su pequeño tamaño lo que los hace enormemente útiles en nuestra área de estudio, ya que la información con la que identificamos un objeto arqueológico se suele reducir a tres descriptores: el yacimiento, la unidad arqueológica o nivel y un número secuencial que lo individualiza de cualquier otro objeto y está relacionado con las coordenadas donde se ha recuperado. El uso de etiquetas troqueladas con todos estos tamaños nos permite elegir el del código DM en función del tama- ño del objeto arqueológico que queremos identificar.

Los códigos DM ofrecen diferentes ventajas respecto al etiquetado tradicional o frente al uso de etiquetas de códigos de barras que son de mayor de tamańo y en muchos casos no se pueden aplicar directamente sobre el objeto, por lo que deben ser adheridas a bolsas contenedoras. Contrariamente, debido a sus reducidas dimensiones, los DM se adhieren directamente sobre la superficie del objeto, reduciéndose sustancialmente 
posibles pérdidas o errores durante la manipulación de los mismos, o al intercambiar los códigos de identificación de los contenedores. Adicionalmente, la información contenida puede ser recuperada incluso si parte del código está dañado, ya que estos códigos ofrecen un sistema de restauración de errores que permite recobrar hasta un $25 \%$ de la información. Esto permite la lectura de códigos parcialmente dañados y garantiza la preservación de la información albergada en los mismos.

\section{El catalogado de artefactos}

Tradicionalmente, el catalogado se ha realizado a mano asignando al objeto arqueológico su identificador en el proceso de excavación e inventariándolo posteriormente en el laboratorio. Para el marcaje se ha utilizado tinta china, aplicada con plumilla o bien algunos tipos de tinta indeleble. Esto conlleva escribir el identificador sobre la superficie del objeto, o sobre una capa de esmalte, barniz o incluso pintura blanca (Tipp-Ex ${ }^{\odot}$ ). El marcaje a mano es un proceso repetitivo y mecánico que puede presentar múltiples errores debido a fallos en la numeración o la ilegibilidad de los caracteres. Además, está sujeto a factores como la porosidad de la pieza, el tamaño y la irregularidad de la superficie. Estos elementos afectan a la calidad del marcaje y hacen que los caracteres resulten ilegibles pasado un tiempo. La vida media de estos tipos de marcaje depende de las condiciones de almacenaje, así como de los materiales utilizados.

Como consecuencia de la falta de un procedimiento fiable de identificación de los objetos arqueológicos, una parte importante del trabajo en museos consiste en repetir el marcaje de los mismos para prevenir la pérdida de información, especialmente en el caso de colecciones relevantes (Dibble et al., 2009). La aplicación de esta nueva técnica para la identificación de los artefactos facilita el almacenaje $\mathrm{y}$ archivo de los restos arqueológicos.

Las infraestructuras que permiten la aplicación de este sistema son el lector de códigos, la impresora, etiquetas troqueladas, el software que las genera y el producto adhesivo (Paraloid B72) que garantiza la preservación del DM adherido a las piezas. La metodología que hemos desarrollado consta de cuatro fases:

\section{Planificación del identificador}

El software que genera las etiquetas permite introducir los atributos relevantes para el cifrado de los códigos, entre los que se encuentran: identificador del yacimiento, nivel arqueológico y número secuencial de catálogo. Estos atributos son transformados en secuencias alfanuméricas (figura 1).

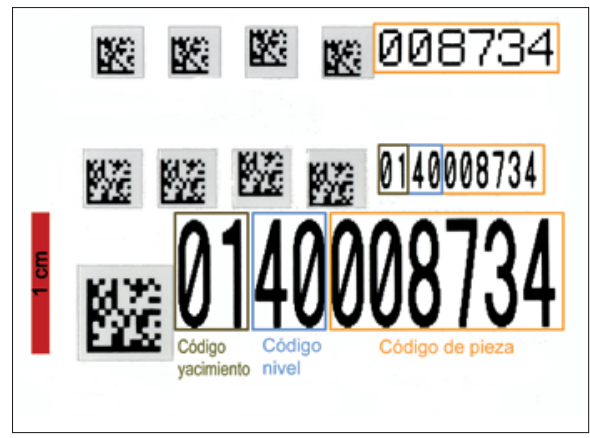

Figura 1. Atributos presentes en las etiquetas troqueladas. 
Estos campos están ordenados jerárquicamente de mayor a menor importancia, siendo el principal el número secuencial de catálogo que se otorga a cada objeto y cuya longitud máxima es de 6 dígitos. Este número no se puede repetir nunca dentro del mismo nivel arqueológico. El segundo atributo es el identificador de nivel y finalmente el código de yacimiento. Cada uno de estos campos cuenta con dos dígitos. La combinación de estos campos conforma series de diez dígitos, suficientes para catalogar cualquier colección independientemente de su tamaño, ya que estas cadenas alfanuméricas, al combinar cifras y letras, dentro de la secuencia multiplican exponencialmente el número de combinaciones posibles.

En función del tamaño del DM seleccionado se puede introducir una secuencia de datos de longitud variable. Así un código de $3,5 \times 3,5 \mathrm{~mm}$, permite almacenar una información máxima compuesta por una cadena alfanumérica de diez dígitos. Si se selecciona trabajar con códigos más pequeños, será preciso seleccionar los campos que se quieren incorporar en la etiqueta.

\section{Impresión etiquetas troqueladas}

Las etiquetas troqueladas están fabricadas con polipropileno, lo que ofrece una durabilidad elevada y las hace muy aptas para la impresión digital, ya que son estables en condiciones variables de temperatura y humedad. Son impresas en tinta térmica con una resolución de 300 dpi (Stevenson, 2009). Este tipo de impresión es una buena solución calidad/precio para la impresión. Actualmente, en el mercado se dispone de una amplia gama de impresoras digitales que permiten la impresión de los códigos sobre las etiquetas troqueladas. Estas etiquetas, al estar diseñadas para este uso, facilitan enormemente el proceso de selección y manipulación de los códigos DM. Hemos de tener en cuenta que el código DM requiere que exista alrededor del mismo un espacio en blanco que facilite su lectura. Al tratarse de una precisión milimétrica, en su impresión repetimos el mismo código cuatro veces sobre cada etiqueta, por lo que el individuo que lleva a cabo el trabajo deberá seleccionar el que reúna las condiciones más precisas en su centrado. Igualmente las propias etiquetas o la resina acrílica que empleamos son fáciles de obtener en empresas especializadas en la distribución de productos de restauración, conservación y almacenaje de material arqueológico.

\section{Adherencia del DM al artefacto}

Las etiquetas son fijadas sobre las piezas mediante Paraloid B72. Esta resina acrílica, usada habitualmente en la restauración y conservación de materiales arqueológicos, ofrece una excelente fijación y conservación de los códigos DM, ofreciendo estabilidad química y resistencia a la decoloración (Koob, 1986; Horie 2005; Podany et al., 2001). La resina acrílica es disuelta en componentes volátiles (acetona o alcohol), lo que facilita su aplicación y secado rápido. Son aplicadas dos capas, una directamente sobre la pieza y posteriormente otra sobre el código DM, lo que permite la fijación de la etiqueta y un buen sellado de la misma. Después de numerosos ensayos hemos establecido que una disolución del Paraloid al $20 \%$ ofrece condiciones óptimas de secado para agilizar el proceso. 
Xavier Roda Gilabert, Sofia C. Samper Carro, Rafael Mora Torcal, Paloma González Marcén, Jorge Martínez Moreno
La aplicación de los códigos Data Matrix (DM) en el registro y la catalogación arqueológica

\section{Lectura y gestión}

Una cuestión importante es la recuperación de la información contenida en el código que puede realizarse por varios sistemas. El método de lectura más habitual es mediante un lector láser, que transfiere directamente los datos del código al ordenador. Este no es el único sistema, ya que pueden captarse por otros soportes digitales como cámaras de vídeo y fotográficas, o incluso cámaras de teléfonos móviles que dispongan de la aplicación que permita su lectura. En nuestro caso, el software de lectura está integrado con el programa de desarrollo propio ArqueoUAB (Mora et al., 2010) que gestiona el inventario de una excavación arqueológica.

\section{Roca del Bous y la Cova Gran de Santa Linya: cinco años de experiencia en el uso de los códigos Data Matrix (DM)}

A lo largo de los últimos cinco años, el catalogado mediante códigos DM se ha aplicado de manera sistemática a los yacimientos excavados desde el Centre d'Estudis del Patrimoni Arqueològic de la Prehistòria de la Universitat Autònoma de Barcelona (CEPAP-UAB), entre ellos destacan la Roca dels Bous y la Cova Gran de Santa Linya (Lleida, España). Ambos yacimientos se sitúan en la zona de contacto entre los valles interiores del Prepirineo y la Depresión del Ebro. Este escenario sugiere que los yacimientos fueron utilizados como refugios por los últimos grupos de neandertales y los humanos modernos que habitaron esta zona. Su característica común consiste en la presencia de múltiples estructuras de combustión, junto con restos óseos y de industria lítica. La abundancia de restos arqueológicos, muchos de pequeño tamaño $(<5 \mathrm{~mm})$ hace de estos dos yacimientos los lugares idóneos para evaluar la viabilidad del uso de los códigos DM en el trabajo de campo y laboratorio (Martínez-Moreno et al., 2011).

Los resultados preliminares de estos estudios se han publicado en revistas especializadas y han introducido elementos de debate dentro de la comunidad científica (entre otros Mora et al., 2008; Mora et al., 2011; Mora et al., 2012; Mora et al., 2014a; Mora et al., 2014b). De forma paralela al desarrollo de los trabajos de investigación básica se ha generado una intensa actividad de divulgación complementaria que, finalmente, ha cristalizado en un programa integral de difusión del patrimonio cultural vinculado a la investigación y al territorio en el cual se localizan los yacimientos (www.larocadelsbous.uab. cat; www.espaiorigens.com).

\section{Del laboratorio al campo}

El primer paso es generar diariamente las etiquetas de los códigos DM que presumiblemente serán usadas durante una jornada de trabajo de campo. A cada artefacto coordenado en el yacimiento se le asigna una etiqueta con el DM que posteriormente le será adherido en el trabajo de laboratorio. Esta rutina obedece a que en los yacimientos no se dispone de red eléctrica, por lo que las impresoras no son funcionales en el yacimiento. De hecho, aparatos como la estación total o las Personal Digital Assistant (PDA) se alimentan de baterías que se recargan diariamente en el laboratorio.

Durante la excavación el procedimiento es relativamente simple. Cuando se coordinan los artefactos, a cada uno se 
les asigna una etiqueta individualizada (figura 2a). En la toma de datos del artefacto, el teodolito láser registra sus coordenadas a las que se les otorga un identificador único e irrepetible que contiene una secuencia de datos conformado por el nivel y número secuencial de catálogo (Parcerisas y Mora, 1995). Esta secuencia alfanumérica es la misma que está impresa en la etiqueta del código Data Matrix. Una vez tomada la posición del artefacto, se introduce en una bolsa de plástico individualizada con su etiqueta correspondiente. Este proceso se agiliza mediante la impresión previa de las etiquetas.

El uso de Personal Digital Assistant (PDA) como cuaderno de campo permite tener un control de los objetos recuperados en campo, ya sean restos líticos, huesos, carbones o semillas, al recoger datos cuantitativos y atributos que califican la contextualización de un artefacto. Las PDA contienen la misma secuencia de códigos que identifica al objeto, por lo que es poco probable que se cometa un error en la identificación individualizada del artefacto (Mora et al., 2010). Este procedimiento implica que, antes de coordinar un artefacto, se ha verificado tres veces la correspondencia entre objeto y código que lo identifica. En primer lugar, la asignación del número secuencia para cada objeto mediante el código DM y posteriormente, la verificación de este número en la estación total y la PDA que ejercen la función de hojas de campo. Este proceso reduce la posibilidad de que se intercambien etiquetas.

Posteriormente, en el laboratorio se vacían los datos del teodolito láser en el ordenador, incorporándolos diariamente a la base de datos. Igualmente, la información que contiene la PDA se vuelca directamente sobre la base de datos, permitiendo la combinación de los datos referidos con la posición espacial y los datos contextuales de cada coordenado recuperado en la excavación (Mora et al., 2001; Mora et al., 2010).
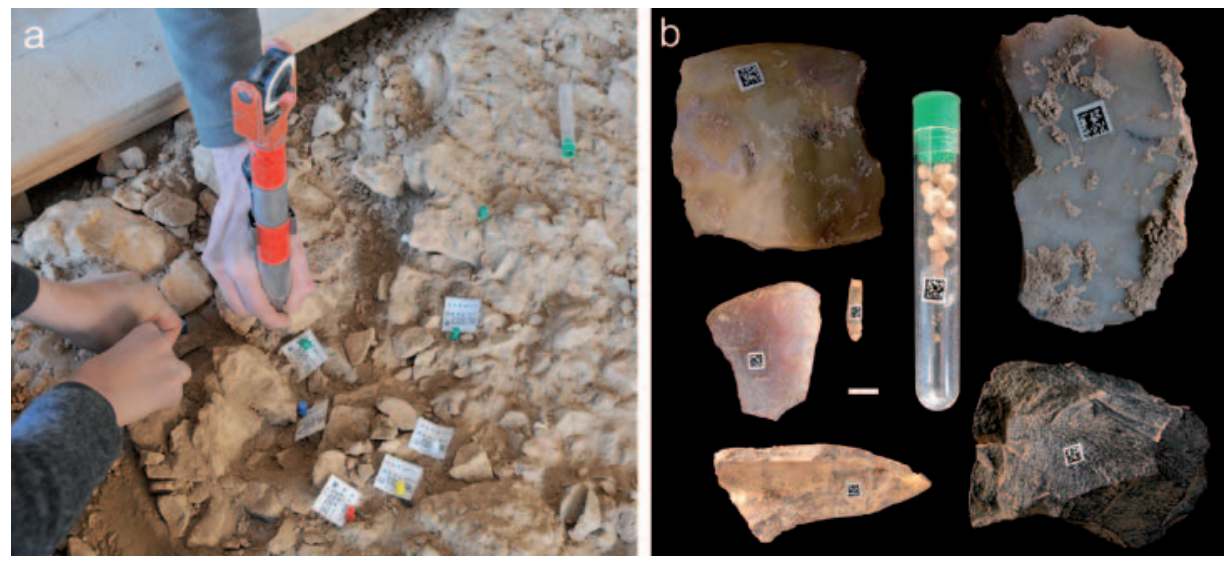

Figura 2. Uso de las etiquetas Data Matrix durante el trabajo de campo. a) Serie de etiquetas junto a piezas en proceso de documentación; b) Ejemplo de piezas sigladas mediante códigos DM. 
Paralelamente, los artefactos son sometidos a tratamientos de limpieza habituales por métodos no agresivos. Una vez limpios, se aplica la primera capa de Paraloid que será la base en la que se colocará el código DM (figura 2b). Cada etiqueta contiene distintos tamaños de códigos y los ejemplares de cada fila contienen la misma información. La elección del tamaño de código se realiza en función de las características del artefacto, especialmente de su tamaño. Al disponer en cada fila de varios códigos, se selecciona aquel que esté mejor centrado dentro del troquelado de la etiqueta. El código se separa de la etiqueta y se adhiere sobre la superficie de la primera capa, y posteriormente se repasa con una segunda capa de Paraloid para proteger la etiqueta.

Hemos señalado que el secado de esta resina es muy rápido, por lo que la pieza puede ser identificada de forma inmediata y segura por medio de la lectura del código DM con el lector láser, al mismo tiempo que es reconocida por la base de datos (figura 3a). Así pueden introducirse otros atributos que no se han registrado durante la excavación, como el peso, la métrica y otras informaciones referidas a su estado de conservación, alteraciones tafonómicas, y en general cualquier atributo de análisis incluido en la base de datos. Esta información permite completar el inventario diario de materiales recuperados en el trabajo de campo (figura $3 \mathrm{~b}$ ).

\section{Valoración y problemáticas}

A lo largo de las últimas campañas de excavación se han catalogado varios miles de piezas usando los DM. A modo de ejemplo, incluimos un gráfico donde se presenta el recuento de las piezas recuperadas en ambos yacimientos (figura 4). En ambos casos la totalidad de las piezas
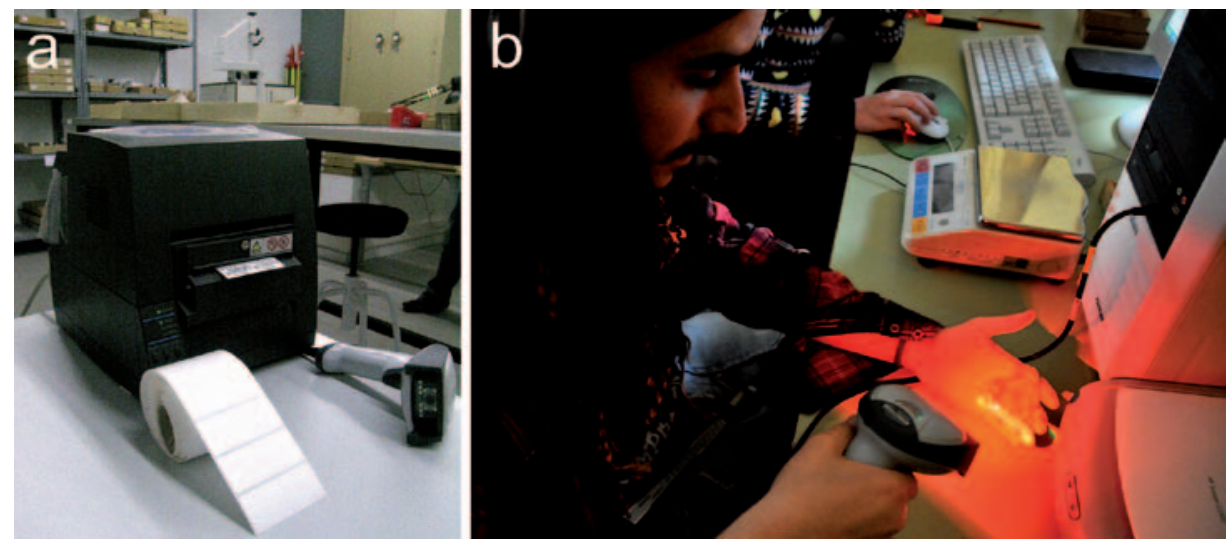

Figura 3. Gestión de las etiquetas Data Matrix en el trabajo de laboratorio. a) dispositivos usados para la impresión y lectura de las etiquetas troqueladas; b) lectura de los códigos en el proceso de inventariado en la base de datos. 
Xavier Roda Gilabert, Sofia C. Samper Carro, Rafael Mora Torcal, Paloma González Marcén, Jorge Martínez Moreno
La aplicación de los códigos Data Matrix (DM) en el registro y la catalogación arqueológica

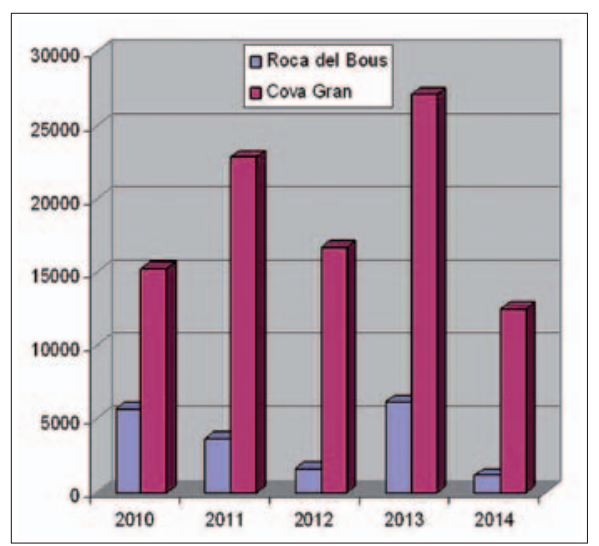

Figura 4. Gráfico con el recuento de las piezas recuperadas en los yacimientos de Roca del Bous y la Cova Gran desde la implantación de los códigos DM.

extraídas fueron identificadas a lo largo de la campaña de excavación dedicando entre dos y tres horas de trabajo de laboratorio diarias.

En ambos yacimientos se han observado cambios en las dinámicas de trabajo que afectan a la productividad y la calidad de los resultados obtenidos en el catalogado. Entre ellos podemos citar una reducción del tiempo empleado en el inventariado, junto con una disminución del personal requerido para el marcaje de los mismos. Por lo tanto, este método es capaz de agilizar el proceso reduciendo además los errores asociados al mismo. En estos momentos las siglas erróneas o pérdidas de información se estiman en porcentajes inferiores al $1 \%$. El porcentaje de errores detectados a lo largo del proceso es minimizado con la revisión sistemática de las piezas. Entre los problemas observados podemos destacar: códigos descentrados (figura 5a), mal adheridos (figura 5c); aparición de burbujas en el Paraloid dificultando la lectura del código (figura 5b), o descamación de la resina (figura $5 \mathrm{~d}$ ). La mayoría de estos problemas tiene su origen en un mal estado del Paraloid aplicado sobre las piezas. Esto es debido a la acumulación de partículas de sedimento en el interior del dispensador o a la evaporación de la acetona por una exposición aeróbica excesiva de la resina. La solución a estos problemas es sencilla y se limita a un control diario del estado del Paraloid, que en condiciones óptimas debe presentar una textura acuosa, y en la aplicación correcta de los DM en cada uno de los pasos del proceso de etiquetado.

\section{Discusión}

Los códigos Data Matrix son una herramienta útil para la gestión de los objetos arqueológicos tanto en el trabajo de campo como en el de gabinete. Además, la introducción de esta tecnología en los museos puede cambiar la forma en la que se gestiona el mantenimiento y conservación de las colecciones.

El objeto arqueológico ha de ser tratado como una entidad a la que se asocia una posición georreferenciada en el espacio y una información contextual. A todo ello se accede a través de su identificador, que es único en la unidad arqueológica a la que se adscribe. El uso de los códigos DM nos permite acceder a la información rápidamente en cualquier momento del proceso del trabajo, ya que la digitalización de la información es algo recurrente hoy día.

En esta valoración debe indicarse que estos sistemas digitales no están sometidos a errores habituales en el marcaje manual, 
en el que no son raros fallos que conllevan la pérdida irreversible de información contextual (Dibble y Mc Pherron, 1998). En la actualidad el uso de los códigos DM se está extendiendo a diferentes ámbitos del patrimonio y la arqueología. Entre ellos podemos citar grupos de investigación como Sustainable Archaeology de
Canadá, que ha implementado el uso de códigos para la gestión de colecciones arqueológicas (Bathurst y Westby, 2013) o el yacimiento de época histórica del Cástulo (Linares, Jaén) (www.estudiospatrimoniales.es).

Otra cuestión pertinente es indicar si la migración desde los sistemas tradicio-

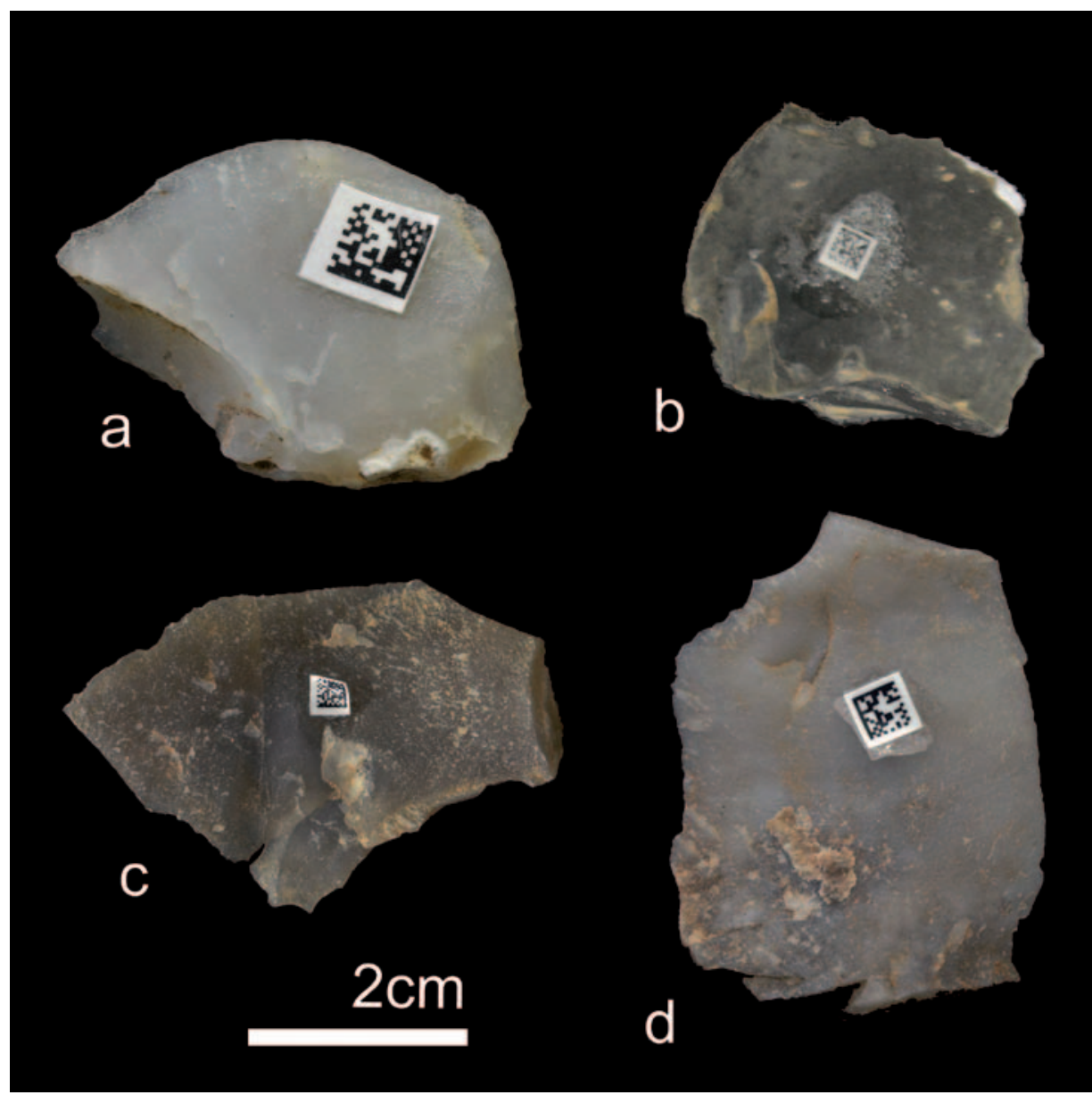

Figura 5. Problemáticas derivadas de la implantación de los códigos Data Matrix. a) códigos descentrados; b) aparición de burbuja; c) mal adheridos; d) descamación de la resina. 
nales de catalogado hacia métodos digitales como los códigos DM es rentable. Esta implementación tecnológica supone una mejora significativa al superar deficiencias derivadas del marcaje manual. Por otro lado, su puesta en práctica supone una inversión económica en la compra de aparatos como lectores láser o impresoras que actualmente no es excesivamente gravosa. El proceso de lectura del código se realiza de forma mecánica mediante lectores láser o aplicaciones gratuitas disponibles para dispositivos móviles.

En lo referido a otras aplicaciones de los DM en la gestión del patrimonio arqueológico, queremos destacar su utilidad en la organización de exposiciones. Los restos originales o réplicas expuestos pueden ser identificados con códigos DM y vinculados a contenidos multimedia. La implementación de sistemas de códigos facilita la creación de contenidos interactivos mediante los cuales el público general puede manipular material arqueológico marcado con códigos DM y ofrecen una herramienta para la difusión del conocimiento a audiencias juveniles, acostumbradas al uso de este tipo de implementos. Los visitantes, mediante la lectura de los códigos, acceden a vídeos, audios u otro tipo de soportes, usando varios tipos de dispositivos de uso cotidiano en la sociedad actual (por ejemplo, tabletas digitales, teléfonos inteligentes, PDA) que implican que los museos no deben hacer una inversión en dispositi- vos para la lectura de códigos (Farina et al., 2014).

\section{Conclusión}

Los resultados obtenidos a lo largo de los últimos cinco años en los yacimientos de Roca dels Bous y la Cova Gran de Santa Linya subrayan una serie de ventajas en el uso de códigos DM para el catalogado de los restos arqueológicos:

- Fiabilidad en la impresión digital de las etiquetas troqueladas que evita el error humano.

- Reducción del tiempo empleado en el inventariado a la vez que se minimizan los errores derivados del mismo.

- Aumento en la productividad y efectividad del proceso de etiquetado que superan el coste económico de las infraestructuras necesarias.

- Durabilidad de los códigos y de la resina acrílica utilizada (Paraloid B72), que hace que este sistema sea óptimo para la gestión y el mantenimiento de grandes colecciones.

- Rapidez en la recuperación de la información contenida en el código que agiliza la manipulación de los objetos.

Bajo esta perspectiva, los códigos DM suponen un importante avance, $y$ deben considerarse una alternativa en la gestión y catalogación de colecciones depositadas en fondos de museos y almacenes arqueológicos. 


\section{Agradecimientos}

Los trabajos de campo han sido subvencionados por el Servei d'Arqueologia i Paleontologia de la Generalitat de Catalunya. Las investigaciones en la Cova Gran y la Roca dels Bous forman parte del proyecto «Poblamiento humano durante el pleistoceno final e inicios del holoceno en el sureste de los Pirineos (HAR2010-15002, HAR201342338)» y del grupo de investigación 2014SGR-0084. Xavier Roda Gilabert es beneficiario de la ayuda FPI-MINECO (BES-2011-045180). Sofía C. Samper Carro es beneficiaria de una beca predoctoral dentro del programa Australian Research Council's Laureate Fellowship (FL120100156).

\section{Referencias bibliográficas}

Bathurst, R.R.; Westby, K. 2013. Digital tools for archaeological collections and inventory management. Poster presented at the annual meeting of the Society for American Archaeology, Honolulu, HI.

Canals, A.; Guerra Rodríguez, D. 2011. «Yacimientos arqueológicos de la Sierra de Atapuerca: Un sistema inalámbrico y computerizado de registro de datos de campo». Virtual Archaeological Review 2(4): 147-50.

Design, C.B. 2010. Technical data sheet Paraloid ${ }^{\circledR}$ B72 Fixative (SY7F).

Dibble, H.; McPherron, S. 1998. "On the computerization of archaeological projects", Journal of Field Archaeology 15, 431-40. <http://dx.doi.org/10.2307/530045>

Dibble, H.L.; Marean, C.W.; Mcpherron, S.P. 2007. "The use of barcodes in excavation projects». The SAA Archaeological Record 7, 33-38.

Dibble, H.; Mcpherron, S.; Sandgathe, D.; Goldberg, P.; Turg, A.; Lenoir, M. 2009. "Context, curation, and bias: an evaluation of the Middle Paleolithic collections of CombeGrenal (France)». Journal of Archaeological Science 36, 2540-2550.

Farina, S.; Paganucci, S.; Landini, W. 2011. «Data Matrix Codes: Esperimental use in a Museum Exhibition». Atti Soc, Tosc. Sci. Natu. Mem. Serie B, 118: 75-80.

Коов, S. 1986. "The use of Paraloid B-72 as an adhesive: its application for archaeological ceramics and other materials». Studies in Conservation 31, 7-14. $<$ http://dx.doi.org/10.1179/sic.1986.31.1.7>

Horie, C. 2005. Materials for Conservation. Organic Consolidants, Adhesives and Coatings, Elsevier.

Laplace, G. 1971. «De l'application des coordonnés à la fouille stratigraphique». Munibe (Antropología y Arqueología) 2-3: 19-71.

Martínez-Moreno, J. I.; De La Torre, R.; Mora, R.;. Casanova, J. 2010. «Technical variability and changes in the pattern of settlement at Roca dels Bous (Southeastern Prepyrenees, Spain)», 1-23.

Mora, R.; Parcerisas, J.; Martínez, J. 2001. "Computer-based recording systems of Pleistocene deposits with large mammals». En: Cavarretta, G.; Gioia, P.; Mussi, M.; Palombo, M.R. (Eds.), The World of Elephants, Roma, 219-23. 
Mora, R.; Martínez-Moreno, J.; Casanova, J. 2008. «Abordando la noción de "variabilidad musteriense" en Roca dels Bous (Prepirineo suroriental, Lleida)». Trabajos de Prehistoria 65, 13-28.

Mora Torcal, R. A.; Benito-Calvo, J.; Martínez-Moreno, I.; De la Torre, S.; Vega Bolívar, M.; Roy, X.; Roda Gilabert, A.; Samper, S. 2014. "Una secuencia clave en la Prehistoria del Mediterráneo Occidental: Cova Gran de Santa Linya (Prepirineo de Lleida)». En: Sala Ramos, R.; Carbonell, E.; Bermúdez de Castro, J. M.; Arsuaga, J. L. Los cazadores recolectores del Pleistoceno y del Holoceno en Iberia y el estrecho de Gibraltar. Estado actual del conocimiento del registro arqueológico. Burgos: Universidad de Burgos/Fundación Atapuerca, 162-66.

Mora Torcal, R.; Martínez-Moreno, J.; Benito Calvo, A.; Roy Sunyer, M.; Roda Gilabert, X.; Casanova Martí, J.; Torresáinz, I. D. L. 2012. «Roca dels Bous y Cova Gran: historias en torno a dos abrigos musterienses del Prepirineo de Lleida». En: Fullola Pericot, J.M.; Ferrer Palma, J. E. Neanderthales en Iberia: Últimos avances en la investigación del Paleolítico Medio Ibérico. Málaga: Servicio de Publicaciones/Centro de Ediciones de la Diputación de Málaga. XXXIII: 101-126.

Mora, R.; Benito-Calvo, A.; Martínez-Moreno, J.; Gonzalez Marcen, P.; De la Torre, I. 2011. "Chrono-stratigraphy of the Upper Pleistocene and Holocene archaeological sequence in Cova Gran (south-eastern Pre-Pyrenees, Iberian Peninsula)». Journal of Quaternary Science 26(6): 635-44. $<$ http://dx.doi.org/10.1002/jqs.1486>

Mora, R.; Martínez-Moreno, J.; De la Torre, I. 2010. «ArqueoUAB: a systematic archaeographic for the analysis of Palaeolithic sites». En: Melero, P.; Cano, P.; Revelles, J. (Ed.), Fusion of cultures, Granada, Spain, April 6-9, 2010, 125-28.

Mora, R.; Martínez-Moreno, J.; Roda Gilabert, X.; De la Torre, I.; Benito-Calvo, A.; Roy, M.; Samper, S.; Vega, S.; Pizarro, J.; Plasencia, J. 2014. «El yacimiento Musteriense de la Roca dels Bous (Prepirineo de Lleida)». En: Sala Ramos, R.; Carbonell, E.; Bermúdez de Castro, J. M.; Arsuaga, J. L. Los cazadores recolectores del Pleistoceno y del Holoceno en Iberia y el estrecho de Gibraltar. Estado actual del conocimiento del registro arqueológico. Burgos: Universidad de Burgos/Fundación Atapuerca: 159-62.

Martínez-Moreno, J.; González Marcén, P.; Mora Torcal, R. 2011. «Data matrix (DM) codes: A technological process for the management of the archaeological record». Journal of Cultural Heritage 12(2): 134-39. $<$ http://dx.doi.org/10.1016/j.culher.2010.10.001>

Parcerisas, J.; Mora, R. 1995. «La estación total inteligente y sus aplicaciones en el trabajo arqueológico». En: Valdés, L.A., I.; Pujuana, I. (Ed.). Aplicaciones informáticas en Arqueologia: teorias, y sistemas. Bilbao, 409-17.

Podany, J.; Garland, K.M.; Freeman, E.R.; Rogers, J. 2001. «Paraloid B-72 as a structural adhesive and as a barrier within structural adhesive bonds: evaluations of strength and reversibility". Journal of the American Institute for Conservation 40, 15-33. <http://dx.doi.org/10.1179/019713601806113120>

Renfrew, C.; Bahn, P. 1991. Archaeology. Theories, methods and practice. Londres: Thames and Hudson Ltd.

Stevenson, R. 2005. Láser Marking Matrix Codes on PCBs Printed Circuit Design and Manufacture.

Wikipedia. 2014. Data Matrix. <http://en.wikipedia.org/wiki/Data_Matrix> 
